Cinémas

Revue d'études cinématographiques

Journal of Film Studies

\title{
Les lois de prolongement et leurs effets sur la mise en scène de l'espace cinématographique
}

\section{Jun Sato}

Volume 9, numéro 1, automne 1998

Les Dispositifs de médiation au cinéma

URI : https://id.erudit.org/iderudit/024774ar

DOI : https://doi.org/10.7202/024774ar

Aller au sommaire du numéro

Éditeur(s)

Cinémas

ISSN

1181-6945 (imprimé)

1705-6500 (numérique)

Découvrir la revue

Citer cet article

Sato, J. (1998). Les lois de prolongement et leurs effets sur la mise en scène de l'espace cinématographique. Cinémas, 9(1), 79-93.

https://doi.org/10.7202/024774ar
Résumé de l'article

Cet article constitue un exposé des trois lois de prolongement du hors-champ, du hors-plan et de l'action. Fondées sur la perception et la cognition des spectateurs, ces lois deviennent des dispositifs de la mise en scène cinématographique. Grâce aux effets de prolongement, le spectateur infère naturellement une image potentielle à partir de ce qui est réellement montré. Assurant la collaboration du spectateur, ces lois garantissent au réalisateur certaines libertés lors du tournage; elles permettent notamment de construire une organisation spatio-temporelle reposant sur un montage discontinu et fragmenté. Ces lois sont applicables, quel que soit le genre de cinéma, en fiction ou en documentaire. 


\title{
Les lois de prolongement et leurs effets sur la mise en scène de l'espace cinématographique
}

\author{
Jun Sato
}

\begin{abstract}
RÉSUMÉ
Cet article constitue un exposé des trois lois de prolongement du hors-champ, du hors-plan et de l'action. Fondées sur la perception et la cognition des spectateurs, ces lois deviennent des dispositifs de la mise en scène cinématographique. Grâce aux effets de prolongement, le spectateur infere naturellement une image potentielle à partir de ce qui est réellement montré. Assurant la collaboration du spectateur, ces lois garantissent au réalisateur certaines libertés lors du tournage; elles permettent notamment de construire une organisation spatio-temporelle reposant sur un montage discontinu et fragmenté. Ces lois sont applicables, quel que soit le genre de cinéma, en fiction ou en documentaire.
\end{abstract}

\section{ABSTRACT}

This article examines the three laws of extension: "out of frame," "out of shot," and action. Based on the spectator's cognition, these laws become apparatuses of cinematographic direction. Thanks to the effects of extension, the spectator naturally infers a potential image based on what is actually shown. By assuring the collaboration of the spectator, these laws guarantee the director certain liberties in filming. In particular, they allow the construction of a spatio-temporal organization founded on discontinuous, fragmented montage. Theses laws are applicable regardless of genre in both fiction and documentary cinema. 
Le cinéma ressemble au monde réel (l'analogie), mais l'une des particularités de ce mode d'expression est la double limitation brusque de l'espace et du temps. On appelle espace du champ (visuel ${ }^{\text {') }}$ tout ce qui est montré à l'intérieur de cette limite spatiale, temps du plan ${ }^{2}$ tout ce qui est à l'intérieur de la limite temporelle, c'est-à-dire de la durée enregistrée. On traite d'habitude séparément les deux aspects, spatial et temporel, mais on pourrait dire que cette étendue constitue une unité spatio-temporelle, considérée comme un continuum de quatre dimensions (trois dimensions spatiales et une dimension temporelle). Le cinéaste peut choisir ces unités spatio-temporelles lors du tournage et les mettre en ordre plus ou moins librement lors du montage. Le dispositif instrumental qui découpe cette unité s'appelle la caméra. L'acte de découper: filmer.

Il existe deux types d'espaces au cinéma: l'espace du champ et l'espace hors champ, comme l'a bien spécifié Noël Burch dans Praxis du cinéma (p. 39). Mais l'espace hors champ n'étant pas visible, on peut donc le considérer comme un espace virtuel ou potentiel. Si l'on admet une symétrie entre l'espace et le temps, il existera un temps hors plan par opposition au temps du plan. Le temps hors plan est également invisible, donc virtuel. Toutefois, la virtualité ne signifie pas une totale liberté imaginaire du spectateur, bien qu'elle appartienne à son monde imaginaire: le hors-champ et le hors-plan sont influencés respectivement par l'espace du champ et le temps du plan.

Dans notre ouvrage De l'espace à l'écran, nous avons tenté de mettre en évidence les bases sur lesquelles reposait cette virtualité. Elles consistent dans la double continuité de l'espace et du temps et ont pour résultat ce que nous avons proposé d'appeler l'effet de prolongement. Il se manifeste sous trois formes, assimilables à trois lois: la loi de prolongement hors champ (entre le champ et le hors-champ), la loi de prolongement hors plan (entre le plan et le hors-plan), enfin la loi de prolongement de l'action. Il s'agit de lois psychoscénographiques, car d'une part, elles sont fondées sur la perception ou la cognition des spectateurs; d'autre part, elles sont le fondement de règles générales de mise en scène.

La prise en compte de ces lois permet de mieux comprendre certains principes d'organisation du film au plan de l'analyse et 
d'apporter un appui à la construction des films, sur les plans scénique et scénarique.

Nous donnerons d'abord quelques précisions sur ces trois lois; puis nous traiterons de certaines de leurs applications, au tournage et au montage; enfin, nous aborderons la question de l'organisation de l'espace impliquée par ces lois.

\section{Loi de prolongement hors champ}

La première loi concerne l'aspect spatial du cinéma. Il s'agit de l'appréhension visuelle du passage entre le champ et son hors-champ immédiat: le hors-champ est considéré par tout spectateur comme le prolongement du champ. Cette hypothèse psychologique de la perception et de la cognition est à rapprocher de la loi de "bonne continuation" de la Gestalttheorie, ainsi que de la "loi de permanence de l'objet" découverte par Jean Piaget. On peut également interpréter ce phénomène en termes de relation indicielle, comme l'a fait Françoise Hautreux dans Indice et cinéma documentaire.

Cette loi trouve son application à tous les niveaux, c'est-à-dire non seulement au niveau des formes abstraites (forme géométrique, couleur, matière), mais encore à celui des formes concrètes (ciel, champ de fleurs, foule).

En principe, cette loi consiste en la continuité entre la partie périphérique du champ et la partie immédiatement extérieure de ce même champ dans le hors-champ. Cependant, le prolongement est également influencé par l'ensemble du champ visuel, c'est-à-dire par la manière dont il s'étend, depuis son centre jusqu'aux limites du hors-champ. Par exemple, la succession de traces de pieds, considérée comme une ligne en pointillés, produit l'effet de prolongement jusqu'au hors-champ, malgré la discontinuité des traces. Son influence diminue au fur et à mesure que la partie de l'espace s'éloigne du champ.

Il existe deux types de hors-champ: le hors-champ proprement dit, hors des limites du cadre du champ, et le champ non sensible, inclus dans le champ géométrique. Contrairement au cadre pictural, le cadre cinématographique n'est pas visible en lui-même. Lors de la projection, c'est une ligne imaginaire qui sépare l'espace représenté de celui de la salle de projection, dont 
l'obscurité aide l'effet de prolongement. Lors du tournage, audelà du cadre de la caméra s'étend l'espace filmé.

Il existe une relation analogue entre le champ sensible et le champ non sensible. Dans la plupart des cas, il s'agit d'une relation de masquage partiel d'un espace (masqué partiellement) par un autre espace (masquant). Le contour du masquant est ici équivalent à la ligne du cadre. La partie de l'espace non masquée est prolongée chez le spectateur dans l'espace masqué, au-delà de la ligne de contour de l'espace masquant. L'image virtuelle de l'espace masqué dans le champ non sensible est mentalement superposée à celle de l'espace masquant. Cette fois, l'effet d'interférence peut surgir, notamment lorsque les deux images (la masquante et la partie non masquée) sont semblables. René Magritte a proposé, dans une série de tableaux nommée Condition humaine, un cas extrême: le sujet d'une toile sur un chevalet ressemble à tel point au paysage alentour qu'il crée une confusion totale. On ne peut apercevoir le contour de cette toile. Il n'est pas inutile de répéter que c'est le contour qui importe, et non le rapport entre l'intérieur et l'extérieur. Par exemple, l'effet de prolongement ne joue pas entre l'extérieur et l'intérieur de la maison. On peut également rencontrer l'effet de prolongement lorsque le champ sensible est limité par une partie obscure et pratiquement non sensible de l'espace, provoqué par les conditions de l'éclairage. Kon Ichikawa a constamment utilisé cette mise en scène dans La Vengeance de Yukinojo (1962), en éclairant uniquement une partie centrale du champ par le «spot» lumineux.

\section{Loi de prolongement hors plan}

La loi de prolongement hors-plan concerne l'appréhension visuelle du passage temporel entre la fin (ou le début) du plan et son hors-plan: le plan se prolonge jusqu'au hors-plan. Cette loi est valable, quel que soit le mode d'enchaînement des plans (coupe franche, fondu enchaîné, volet, iris, etc.). Lorsqu'un plan est suivi par un autre plan qui ne consiste pas en un fond uniforme, est possible l'effet d'interférence entre l'image virtuelle du hors-plan et celle du plan suivant. Dans le cas du fondu enchaîné, la superposition des deux images est réelle. 
Bien que l'effet de prolongement diminue progressivement, il peut persister au-delà du plan immédiatement suivant. Autrement dit, il peut être différé. Par exemple, l'utilisation du plan de coupe s'appuie sur cet effet, car le contenu montré du plan de coupe est généralement assez différent de celui des plans entre lesquels il se place, et sa durée est relativement courte. Quand le plan de coupe se termine, l'influence de l'effet de prolongement du plan qui l'a précédé demeure, le spectateur confirmant spontanément la situation initiale. Il en va de même du montage parallèle (ABAB...).

L'effet de prolongement peut se manifester à l'intérieur du même plan. Par exemple, quand on éteint la lumière à l'intérieur du plan, l'image du plan se prolonge dans la partie obscure du plan. Diégétiquement parlant, il s'agit d'un seul plan, mais qui se partage en deux parties de natures très différentes, comme s'il s'agissait de deux plans séparés.

On peut citer deux exemples d'effet de prolongement hors plan à l'intérieur d'un même plan tirés de L'Homme à la caméra (Dziga Vertov, 1929). Les deux scènes peuvent être interprétées de la même manière: 1) le train s'approche de la caméra posée sous les rails, passe au-dessus et s'en va vers l'arrière ; 2) le train se déplace latéralement juste devant la caméra fixe. Si le spectateur fait attention au paysage, le train en passant occupe tout l'écran et fonctionne comme un volet naturel, masquant le paysage. L'image virtuelle du paysage demeure chez le spectateur au moment du passage du train en raison de la loi de prolongement hors plan. Par contre, si le spectateur suit le mouvement du train, ce mouvement est prolongé après son passage en raison de la loi de prolongement de l'action que nous allons exposer.

\section{Loi de prolongement de l'action et types d'apparition ou de disparition}

La troisième loi de prolongement concerne la continuité de l'action. Une action présentée suggère toujours chez le spectateur, jusqu'à preuve du contraire, sa continuation au-delà de sa disparition sur l'image (action anticipée), ou bien son commencement en deçà de son apparition (action rétrospective). 
Cette loi peut sans doute être interprétée à la lumière des deux lois précédentes, puisque l'apparition et la disparition de l'agent de l'action s'effectuent souvent entre le champ et le horschamp, ou le plan et le hors-plan. Toutefois, il nous semble utile de la traiter à part, notamment lorsque l'action est le fil conducteur du film et que l'espace du champ et le temps du plan autour d'elle ne jouent qu'un rôle secondaire. Dans ce cas, le spectateur prête attention à l'action elle-même plutôt qu'à son espace environnant.

Analysons à présent cette loi dans la perspective des types d'apparition et de disparition. Nous nous contenterons ici d'aborder uniquement la disparition. Il existe deux cas typiques de disparition. Dans le premier, l'agent de l'action quitte complètement le champ avant que le plan ne se termine: la loi de prolongement hors champ semble être seule concernée. Dans le second, lorsque le plan se termine, l'agent de l'action demeure présenté en plein milieu du champ: la situation semble pouvoir être interprétée en terme de la loi de prolongement hors plan. Mais il existe un cas, intermédiaire: le plan se termine au moment où l'agent de l'action est en train de disparaître du champ. Il en va presque de même si le plan se termine juste avant l'arrivée de l'agent à la limite du champ, ou juste après sa disparition hors champ. Dans tous ces cas, il s'agit d'un double effet de prolongement de l'action: champ/hors-champ et plan/ hors-plan. Premier effet: le spectateur appréhende l'image virtuelle de l'action dans le hors-champ. Second effet: cette image virtuelle se prolonge encore dans le hors-plan. Cela suggère que le contenu virtuel du hors-plan dépend en partie de l'effet de prolongement hors champ. Peut-être est-ce en raison de ce double effet que la mise en scène de la disparition est fréquemment utilisée en cinéma de fiction.

Il existe un cas particulier de scène d'apparition et de disparition non fondé sur le jeu du champ / hors-champ ou du plan / hors-plan. Il se passe à l'intérieur du champ et du plan: l'agent de l'action, se dirigeant de l'infini vers la caméra, commence à apparaître, tel un point lointain, à l'arrière-plan du champ. Or le spectateur, ne parvenant pas encore à identifier cet agent ni son action, ne peut les prolonger dans un sens plutôt que dans 
un autre, en l'absence d'indices suffisants. Nous en découvrons un exemple dans Laurence d'Arabie (David Lean, 1962), lors de la séquence d'arrivée d'Ali (Omar Sharif). Au milieu du désert, il n'y a rien jusqu'à l'horizon. Un des guides de Laurence lève tout à coup les yeux et regarde un point à l'infini, parfaitement ambigu, auquel le spectateur ne peut appliquer un effet précis de prolongement hors champ et hors plan. Ensuite, un plan subjectif présente une toute petite ligne de poussière à l'horizon et le personnage commence à apparaître. Sa dimension grandit progressivement, suggérant son avancée. De ce fait, le prolongement virtuel de sa progression vers le spectateur devient possible (prolongement de l'action), à supposer que l'on coupe brusquement le plan.

\section{Des effets des lois sur le tournage}

Le spectateur est en grande partie prisonnier de ce qui est montré. Il infère le hors-champ et le hors-plan à partir de ce que lui offre le champ. Quant au cinéaste, tirant partie de la loi de prolongement hors champ, il peut occulter en toute tranquillité ce qui gêne pour le tournage: le dispositif cinématographique (micros, magnétophones, appareils d'éclairage, etc.), l'équipe de tournage et les coulisses du plateau. Il peut également, grâce à la loi de prolongement hors plan ou de prolongement de l'action, organiser d'une manière plus ou moins libre le temps d'enregistrement de la prise de vue et interrompre celle-ci au bon moment.

En ce qui concerne le film de fiction, la mise en scène de l'espace et du temps est relativement libre lors du tournage. On peut ainsi éviter de fabriquer des éléments de décor du plateau (plafond, quatrième mur, etc.) dont le spectateur infere spontanément la présence - sinon la forme - grâce à la loi de prolongement hors champ. On peut également fragmenter la scène en plusieurs plans et tourner ceux-ci dans un ordre libre. On peut enfin répéter plusieurs prises d'un même plan, car ce qui importe est l'enchaînement d'une même action fondé sur l'effet de prolongement de l'action. Comme on le verra plus tard, un tel effet résulte du montage.

Les lois de prolongement sont également utiles lors du tournage d'un film documentaire, dont la mise en scène tend à se 
rapprocher le plus possible de l'auto-mise en scène ${ }^{3}$ de l'espace et de l'action observés, mais se trouve soumise à diverses contraintes. Malgré ces contraintes, le cinéaste a bien souvent la possibilité de découvrir un point de vue satisfaisant, en associant un angle de vue, un cadrage et un poste d'observation, qui permettent de chasser les éléments gênants hors champ. Grâce à la loi de prolongement du plan ou de l'action, il lui est aisé d'interrompre la prise de vues pour changer le cadrage ou économiser le temps, à condition de ne pas occulter des moments importants. Si un tel risque est encouru, le cinéaste est contraint de prolonger l'enregistrement. S'il souhaite changer le cadrage, il doit déplacer en continuité le poste d'observation, qui devient alors itinérant ${ }^{4}$.

Dans Qu'est-ce que le cinéma?, André Bazin a critiqué la mise en scène de la fameuse séquence de la chasse au phoque de Nanouk of the North (Flaherty, 1922), qui ne montre pas simultanément dans le même plan le chasseur, le trou et le phoque (p. 59). Or, si l'on examine de près les images, on voit que le cinéaste a montré en même temps ces trois éléments, y compris la corde, en fin de séquence. Mais la manière dont sont présentées certaines phases de la chasse est insatisfaisante. Tout

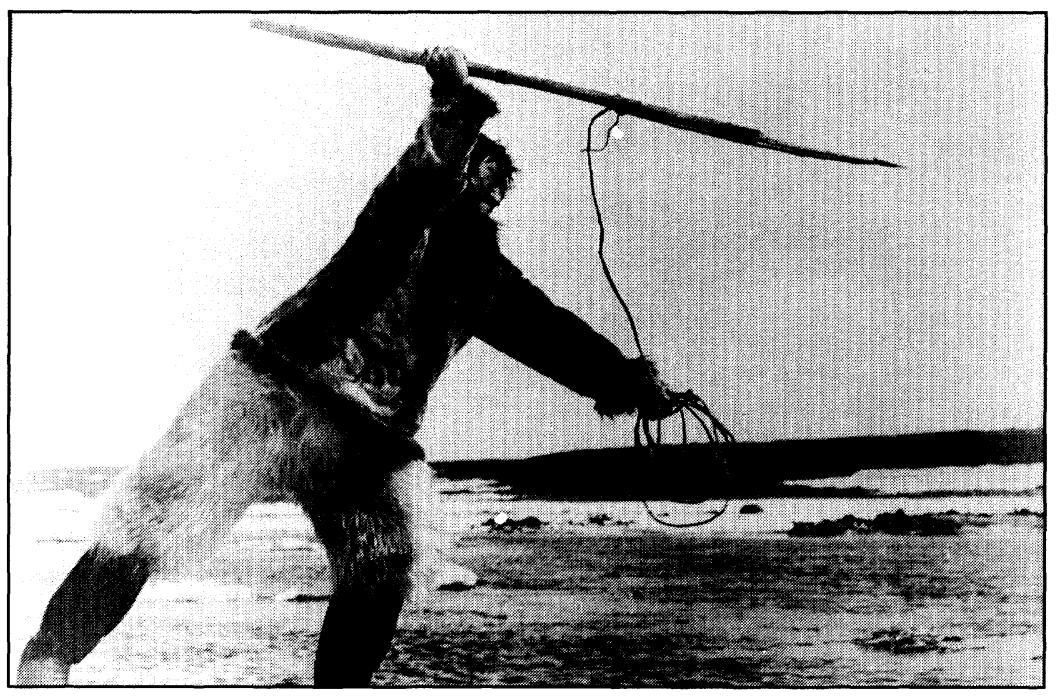

Nanook of the North de Robert Flaherty (1921) 
d'abord, le réalisateur n'a pas directement montré le percement du trou par le chasseur, mais seulement le mouvement du bras vers le bas pour lancer le harpon: quand le plan s'arrête, la pointe du harpon est déjà hors champ. Dans le plan suivant, le trou est déjà percé, la corde semble attachée à un phoque, encore invisible, et le manche du harpon est détaché. Le moment décisif de cette phase n'est indiqué que par la liaison des deux plans. Est également insuffisante la manière dont est présentée la phase de l'agrandissement du trou pour que le phoque y passe; plus tard n'est pas non plus montré le moment précis où le phoque apparaît par le trou. Les moments stratégiques de la chasse sont suggérés par l'effet des lois de prolongement. Mais cela demeure nettement insuffisant dans le cas d'un film qui se veut en grande partie documentaire.

\section{Quelques conséquences sur le montage}

Tout plan, quel qu'il soit, peut être soumis à l'effet de prolongement hors plan en son début et à sa fin. S'il existe une action dans ce plan, l'effet de prolongement de l'action le concerne également. Même l'effet de prolongement hors champ peut servir à tout instant au déroulement du plan et lors de sa transition avec un autre plan. Les effets de prolongement fonctionnent comme une colle imaginaire pour relier deux plans. En principe, on peut relier un quelconque plan à un autre, mais le résultat n'est pas toujours harmonieux (c'est toute la différence entre le raccord et le faux raccord, artificiel). L'aspect naturel du raccord dépend en partie du bon effet du prolongement.

Dans Praxis du cinéma, Noël Burch a classé d'une manière systématique les types de liaison entre deux plans consécutifs (p. 21-38). C'est ainsi qu'il a relevé la continuité, la discontinuité définie, la discontinuité indéfinie, le retour en arrière bref, le retour en arrière long. La catégorisation est intéressante, à ceci près qu'il n'est pas toujours possible de vérifier avec exactitude une liaison en continuité. Un indice très précis est indispensable, aidé par l'effet des lois de prolongement. Tel est notamment le cas lorsque l'action est répétitive, comme par exemple, dans une scène de danse, où chaque action n'étant pas identifiable comme telle, la continuité entre deux plans devient équivoque. 
En général, grâce aux influences mutuelles des effets de prolongement du plan (hors plan) et de l'action entre deux plans consécutifs, tout montage tend à donner l'impression d'une continuité dans le temps diégétique, en dépit parfois d'un peu d'incertitude. Certains éléments ou indices renforcent cet effet. Il en va ainsi du dialogue qui chevauche deux plans en champ/ contrechamp. Le plan de coupe provoque souvent l'effet d'une élasticité du temps qui passe, comme on l'a vu plus haut. Le laps de temps que dure ce plan, ou la coupure qui le précède ou le suit, se trouvent diminués, et les deux plans quasi identiques qui l'entourent sont liés par la loi de prolongement hors plan.

L'un des artifices du cinéma est de contracter le temps en dépit de l'impression de continuité que donne la succession des plans. Ainsi, dans Le Goût du saké (Ozu, 1962), exemple déjà cité dans De l'espace à l'écran, au bout d'une séquence de quelques petites minutes dans un restaurant populaire, le soir tombe, alors quil faisait plein jour en début de séquence. $\mathrm{La}$ continuité, malgré chaque changement de plan, est assurée par la loi de prolongement hors plan, renforcée par la continuité du dialogue. C'est à la fin de cette séquence que le spectateur a le sentiment d'un effet curieux.

Parmi les liaisons entre deux plans consécutifs, un cas intéressant est le bref retour en arrière par le second plan. Cette micromise en scène est un peu démodée, mais Eisenstein l'a beaucoup utilisée. Dans Le Cuirassé Potemkine (1925), la scène de la chute d'un commandant est composée de plusieurs plans variant les angles de vue sur la chute. Chaque fois que le plan change, le plan suivant reprend l'action un peu en arrière. Presque invisible, projeté à la vitesse normale, il tend toutefois à souligner l'action. La continuité de la chute est suggérée par la loi de prolongement de l'action cependant que la loi de prolongement hors plan rend sensible le retour en arrière et la position du chuteur par rapport à son milieu.

\section{Lois de prolongement et organisation de l'espace}

Il existe trois manières de décrire l'organisation de l'espace simultanée (présentation des éléments dans un même champ visuel en plan fixe); successive continue (présentation en un seul 
plan mobile par un trajet tel que panoramique, travelling, etc.); successive discontinue (présentation en plusieurs plans fixes ou mobiles). Les deux premières manières montrent plus directement les relations des éléments à l'intérieur de l'espace, mais certaines contraintes relatives au milieu filmé empêchent quelquefois de les utiliser.

C'est dans le cas de la dernière manière, fondée sur l'usage de plusieurs plans, que les lois de prolongement jouent un rôle prépondérant. Plusieurs stratégies de mise en scène sont, dans ce cas, possibles. Nous nous contenterons de citer les trois principales d'entre elles. La première stratégie s'appuie sur l'homogénéité des éléments de l'espace, ou bien sur les traits caractéristiques de cet espace, mais sans indices précis. Dans nos propres expériences de mise en scène - Forum des Halles 1 (1980) et Promenade aux Halles (1985) —, nous avons parfois juxtaposé plusieurs plans montrant chacun des aspects différents de l'espace. Cependant, chaque plan présente également des traits communs de cet espace: matière de verre, utilisation des lignes droites ou hyperboliques dans la structure architecturale. Un aspect particulier (banc, pilier, etc.) montré dans un plan semble s'étendre dans une zone plus vaste, comme le suggère la loi de prolongement hors champ; de même, plusieurs aspects différents de l'espace se réunissent en vertu de la loi de prolongement hors plan, et grâce à leur présentation immédiatement successive.

La deuxième stratégie consiste en l'utilisation d'un élément commun à deux ou à plusieurs plans. Les plans sont logiquement reliés par cet élément, mais une telle liaison est également renforcée par l'effet de prolongement du plan, en vertu duquel le contenu de ce même plan demeure virtuellement chez le spectateur après son arrêt. Lorsque le spectateur aperçoit de nouveau cet élément dans le plan suivant, il associe immédiatement la partie de l'espace montrée dans le premier plan et celle dans le deuxième plan: alors les deux parties de l'espace sont réunies. On notera que pour montrer cet élément commun de manière successive, il est préférable d'éviter, lors de la transition, l'effet de saut dans l'espace de ce même élément par rapport au cadre (brusque changement de position d'un côté à l'autre de l'écran). Il n'est pas nécessaire de 
placer consécutivement deux plans possédant un élément commun, dans la mesure où l'effet de prolongement du plan demeure, même faiblement, sous une forme différée, grâce au mécanisme logico-perceptif à l'œuvre chez tout spectateur.

La troisième stratégie est fondée sur l'action, cas particulier d'un élément commun, mais mobile. L'effet de prolongement de l'action relie un plan à un autre; différentes parties de l'espace accompagnant cette même action sont donc également réunies. Ainsi sont reliées successivement plusieurs parties de l'espace, à partir d'un même agent de l'action. L'intérêt de cette stratégie consiste à réunir sans difficulté des éléments hétérogènes d'un espace, parfois très vaste. Elle permet aussi de créer un espace tout à fait imaginaire au gré du cinéaste. Ce principe fut déjà expérimenté par Koulechov en $1920^{5}$.

Prises dans leur ensemble, ces trois stratégies sont utiles à une présentation économique de l'espace. Elles tendent notamment à mettre l'accent sur les éléments qui constituent l'espace filmique (relation de pure composition). Par contre, elles ne permettent pas toujours de bien exprimer certaines relations internes de l'espace, telles que l'ordre spatial (mode de distribution des éléments) ou encore l'articulation spatiale (mode d'enchaînement des éléments ${ }^{6}$ ).

L'ordre de présentation de deux plans est souvent permutable, mais quelquefois l'effet n'est pas identique, selon qu'est présenté l'ordre $\mathrm{AB}$ ou l'ordre $\mathrm{BA}$. Tel est par exemple le cas entre une partie et le tout (relation métonymique). Si l'on présente tout d'abord l'espace dans son ensemble, puis une partie de cet espace, le hors-champ du second plan est déjà présenté. Aucune information visuelle n'est ajoutée à celui-ci. Dans le cas inverse (présentation de la partie, puis du tout), le hors-champ est évoqué par l'effet de la loi de prolongement du champ jusqu'à la fin du premier plan. Lorsque le premier plan est remplacé par le second, le spectateur vérifie ce qu'il vient d'imaginer ou constate qu'il est trahi par une nouvelle image. Ainsi, dans un gros plan de la jeune protagoniste de Zazie dans le métro (Louis Malle, 1960), on voit le carrelage du métro à l'arrière-plan. Cela laisse supposer au spectateur que Zazie découvre enfin le métro qu'elle s'impatientait de visiter. Or, dans le plan suivant, plus large, on 


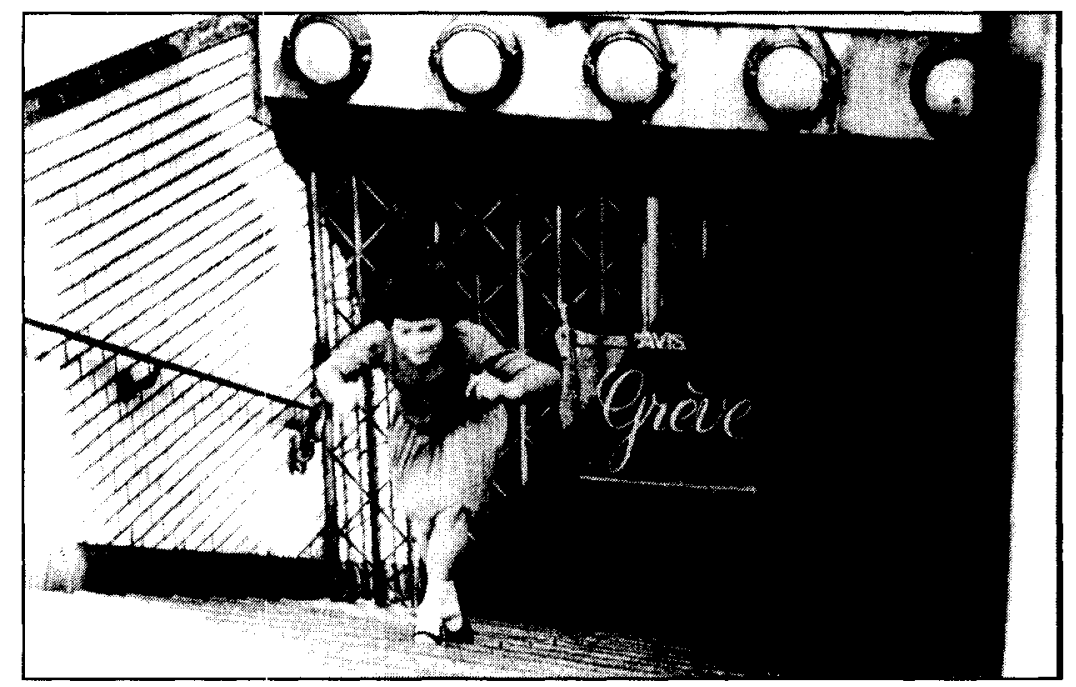

\section{Zazie dans le métro de Louis Malle (1960)}

Collection Cinémathèque québécoise

comprend qu'il s'agit de la partie du mur d'un cabinet de toilette, dans un appartement. Cela relève du gag entièrement visuel, dont les mécanismes doivent beaucoup à la transgression des lois de prolongement.

Nous n'avons abordé ici que l'espace protagoniste, ou l'espace pour lui-même. Mais les lois de prolongement peuvent être également appliquées à l'espace pragmatique, ou espace de l'action. Nous attarder sur ce point déborderait le cadre du présent texte.

L'étude des lois de prolongement et de leurs multiples effets est à peine amorcée ici. Mais nous serions heureux si nos remarques pouvaient être utiles à la réflexion des spécialistes du cinéma.

Université de Paris X

\section{NOTES}

1 Champ: espace délimité par l'appareil d'enregistrement. Il comprend le champ géométrique, déterminé par les caractéristiques de la caméra (champ visuel) et du magnétophone (charnp sonore) et le champ sensible, formé par l'ensemble des objets dont le spectateur peut appréhender les manifestations visuelles ou sonores. Champ visuel: espace délimité par l'objectif de la caméra. Le champ visuel géométrique a la

Les lois de prolongement et leurs effets sur la mise en scène de l'espace cinématographique 
forme d'une pyramide tronquée au niveau du cadre de l'image. Le champ visuel sensible a une forme variable, certains aspects ou objets pouvant se trouver dans le champ visuel géométrique sans pour autant figurer dans le champ visuel sensible du fait qu'ils sont masqués par des obstacles. Ces définitions sont données par Xavier de France dans son ouvrage, Éléments de scénographie du cinéma (Nanterre: Université de Paris X-FRC, 1989, p. 166-167).

2 La définition du plan est ici celle du "plan durée", et non celle de "l'échelle des plans». Il s'agit de la portion de film impressionnée par un seul enregistrement, entre la mise en marche et l'arrêt de la caméra. Toutefois, on pourrait considérer comme un plan, au niveau écranique, une partie de la présentation qui donne l'impression d'être effectuée par un seul enregistrement, tel le moment d'une scène filmée image par image, ou encore la continuité d'un décor, malgré la disparition brusque d'un personnage, comme dans les trucages de Méliès.

3 Auto-mise en scène: notion essentielle en cinématographie documentaire, qui désigne les diverses manières dont le procès observé se présente de lui-même au cinéaste dans l'espace et dans le temps. Cette mise en scène propre, autonome, en vertu de laquelle les personnes filmées montrent de façon plus ou moins ostensible, ou dissimulent à autrui, leurs actes et les choses qui les entourent, au cours des activités corporelles, matérielles et rituelles, est toutefois partiellement dépendante de la présence du cinéaste. La notion d'auto-mise en scène est proposé par Claudine de France dans Cinéma et anthropologie (Paris: Maison des sciences de l'homme, 1982).

4 La notion de poste d'observation est définie par Jane Guéronet dans Le Geste cinématographique (Nanterre: Université de Paris X-FRC, 1987), notamment dans le cadre du tournage à la main. Le poste d'observation est la position du cinéaste dans l'espace à partir de laquelle s'effectue l'enregistrement cinématographique. C'est à partir de ce point précis de l'espace, constant ou non, que le cinéaste observe le déploiement des aspects et des parties des êtres qu'il filme. Le poste d'observation est "fixe" lorsque le corps du cinéaste reste totalement figé. Il est "sédentaire" lorsque le cinéaste demeure posté en un même endroit, mais exécute un mouvement rotatif ou translatoire sur place. Il est enfin "itinérant" lorsque le cinéaste se déplace.

5 Voir à ce sujet Georges Sadoul, Histoire générale du cinéma, tome 5 (Paris: Denoël, 1975, p. 321).

6 Les relations de composition, d'ordre et d'articulation sont les thèmes centraux de la praxéologie qui étudie des formes de l'action. Voir à ce sujet Claudine de France "L'Analyse praxéologique. Composition, ordre et articulation d'un procès" (Technique $\mathscr{\sigma}$ culture, 1983, p.147-170). Nous avons appliqué la méthode d'analyse praxéologique à l'étude de l'organisation de l'espace cinématographique dans De l'espace à l'écran (La Garenne-Colombes: Espace Européen, 1991).

\section{OUVRAGES CITES}

Bazin, André. Qu'est-ce que le cinéma? Paris: Éditions du Cerf, 1975.

Burch, Noël. Praxis du cinéma. Paris: Gallimard, 1986.

France, Claudine de. Cinéma et anthropologie. Paris: Maison des sciences de l'homme, 1982.

France, Claudine de "L'Analyse praxéologique. Composition, ordre et articulation d'un procès", Technique \& culture (1983), p. 147-170.

France, Xavier de. Éléments de scénographie du cinéma. Nanterre: Université de Paris X-FRC, 1989.

Guéronet, Jane. Le Geste cinématograplique. Nanterre: Université de Paris X-FRC, 1987. 
Guillaume, Paul. La Psychologie de la forme. Paris: Flammarion, 1937.

Hautreux, Françoise. Indice et cinéma documentaire. Nanterre: Université de Paris XFRC, 1988.

Piaget, Jean. Construction du réel chez l'enfant. Neuchâtel: Delachaux et Niestlé, 1950.

Sadoul, Georges. Hi:toire générale du cinéma, tome 5. Paris: Denoël, 1975.

Sato, Jun. De l'espace à l'écran. La Garenne-Colombe: Espace Européen, 1991. 